\title{
Trademarks as a Telecommunications Indicator for Industrial Analysis and Policy
}

\author{
Sandro Mendonça \\ Instituto Universitário de Lisboa (ISCTE-IUL), and BRU (PEst-OE/EGE/UI0315/2011), \\ Dinâmia-CET (ISCTE-IUL), UECE (ISEG-UTL), Portugal \\ sfmeiscte.pt
}

\begin{abstract}
Innovation and economic transformation are difficult phenomena to measure, especially in high-tech fast changing sectors. Dynamic competition is a key driving force behind growth and a complex, multidimensional nature calls for an integrated approach. Recently a research agenda that see trademark as a valuable addition to the economic indicators arsenal has been gaining momentum. This section makes the case for employing trademarks as an indicator for assessing dynamic competition and international competitiveness in the telecommunications equipment and services sector. It considers community trademark data to explore stylized facts and recent trends in the European market, a sophisticated and contested world sales pitch on which this data can be employed to generate substantive academic insights as well as useful knowledge for policy-makers at the EU and national levels.
\end{abstract}

Keywords: telecommunications, indicators, trademarks, competitiveness, policy Lisboa, Portugal.

\section{Introduction}

Today's economy is increasingly a communication-intensive world trade system. Competition isn't just about new technology and better service. It is also a fierce struggle for the consumer's attention in an increasingly overwhelmed information environment. Trademark data can constitute a source for fresh insights on how companies and countries are being able to put their message across in the contemporary innovation-driven global economy.

As Schumpeter suggested innovation is about introducing new or improved goods and services into the market, launching new or improved manufacturing and service delivery systems into economic activity, discovering and fulfilling all together new or modified needs and desires [1]. Innovation is then about converting relevant technical knowledge into working useful products. Yet, while an increasing quantity of research has been invested into the study of the sources and effects of innovative operations, it continues to operate on the basis of narrow empirical resources. Dynamic competition is a complex, multidimensional phenomenon. At the same time innovation and industrial transformation are difficult to measure. Engaging with real phenomenon of competition, as opposed to standard textbook accounts of neoclassical competition, is 
an important task since it is a key driving force behind economic growth. This brief note attempts to highlight the potential of this new indicator in the context of applied telecommunications economic analysis.

\section{Mapping and Measuring the Commercial Creativity of Firms and Nations}

Two intellectual property rights share a great deal: patents and trademarks. They yield precious macroeconomic data. Both are "back office" measures of how a country is succeeding in the structural transition to a knowledge-based economy. Both are correlated with innovation performance and provide an insight into ongoing processes of industrial change. Patents are assigned to original, non-trivial and productive inventions. Trademarks such as brand names and logos are solicited by firms to distinguish and protect the reputation of goods, services and their corporate identities. These indicators are also complementary: patent counts are a pointer of technological expertise and trademark statistics are an indicator of commercial capability.

Patents and trademarks are here understood as economic indicators of innovation and industrial change. Numbers of applications of these intellectual property rights allow for complementary readings of the state and evolution of an economy. One key difference sets them apart: trademark data is a much less exploited information resource. Patents have been used for a number of decades now and are commonplace in standard economic benchmarking publications, such as those from the OECD, whereas trademarks have been much less used in applied analysis. They offer the possibility of yielding fresh insights in the process of transformation and competition among modern.

The business of branding goods and services has been an ordinary part of economic life since time immemorial. Symbols were used to keep track the origin of bricks used in Roman constructions, familiar names where used by medieval artisans to distinguish the products of their craft, calves roaming on the western prairies were marked by ranchers to identify their property. However, as a source of empirical insights trademarks have been neglected; in spite that branded goods have become a distinguishing feature of economic life in the twentieth century [2]. This neglect has co-existed, nonetheless, with an increasing awareness in the social sciences that branding (or trademarking) has been emerging as a particular phenomenon worth of explanatory work (e.g., in sociology, psychology), but also of prescriptive consideration (e.g., management studies).

Only very recently, applied economic literature has shown that branding activity may be also captured by trademark applications for the analytical purpose of assessing and monitoring ordinary and extraordinary economic life: trademarks have been shown to provide consistent indications innovation behavior and industrial change. Trademark statistics are interesting because they are $i$ ) increasingly available on electronic platforms, ii) regular long-term data availability, iii) broken down by product classes, $i v$ ) able to capture service and SME innovation, and $v$ ) close to commercialization of new products.

Trademarks are exclusive rights to distinctive words, symbols, shapes, or even smells and holograms. The legal framework of this IPR has been evolving. 
A milestone was the Paris International Convention for the Protection of Industrial Property of 1883 (known as the "Paris Convention') covering patents, trademarks, and designs [2].

\section{Community Trademarks for Competitiveness Analysis}

Marks and other distinctive signs (such as logos, slogans, three-dimensional marks objects) they are commercial signatures that distinguish the products and their producers on the marketplace. The flow of trademarks applications reveals the initiative of creating presence and recognition in targeted commercial arenas. With necessary methodology care data on this activity may be used to build a better understanding concerning rhythms and directions of development of national economies, for instance, in the European context.

One place to look is indeed Europe. With almost 500 million consumers and arguably one the most attractive, and sophisticate markets of the world, this is an especially interesting arena to analyze. Thanks to the Community Trade Mark (CTM), an IPR tool that covers the EU territory and which came into existence in 1996, we can observe trends as they develop. In this section we use for the EU-15 countries' applications obtained from the Office for Harmonization in the Internal Market (OHIM), which is responsible institution for managing CTMs. This European right can be seen as a success story seems it appears very clearly to have provided an answer to a latent demand for this kind of European-wide intellectual property right. The last year in record, 2007, is a special one because the volume of applications became more twice of the level of the launch year, 1996, for the first time [2].

Figure 1 shows absolute numbers for total applications from all EU and non-EU countries since the creation of the launch of the CTM. The first year should be regarded as an abnormal year since intentions to trademark were being accumulated for at least two years before. The local peak noticed around the year 2000 can be associated to the "New Economy" bubble, since trademarks covering high-tech categories where driving growth; only by 2005 aggregate numbers surpass those of the year 2000. By the end of the decade, though, the effects of the so-called "sub-prime crisis"/"great recession"/"little depression" are visible.

\section{Trends in Communication-Intensive Knowledge Economy}

A total of 250,000 trademarks were submitted for the whole territory of the EU during last year. Applications by EU-based firms and organizations constitute around two thirds of this figure. Within Europe Germany has been by far the country originating the largest number of trademarks, a share of a quarter of total applications among the 27 EU Member States. Figure 2, which depicts absolute numbers of new CTMs requested by the most important countries in terms of volume of applications, shows a clear trend for the last decade. There is a marked and generalized movement towards intensified trademark deployment. 


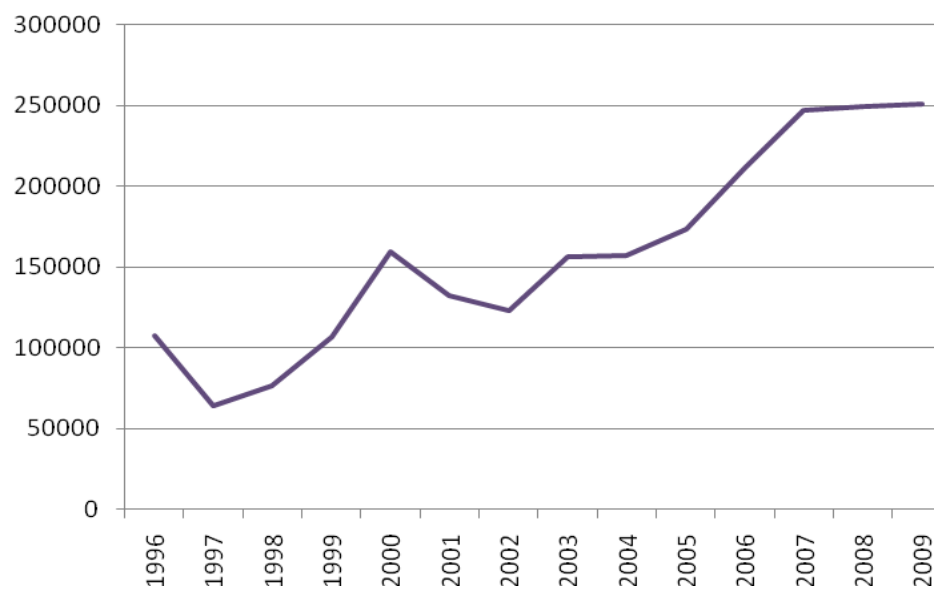

Fig. 1. Total CTM applications, 1996-2009 [3] ${ }^{1}$

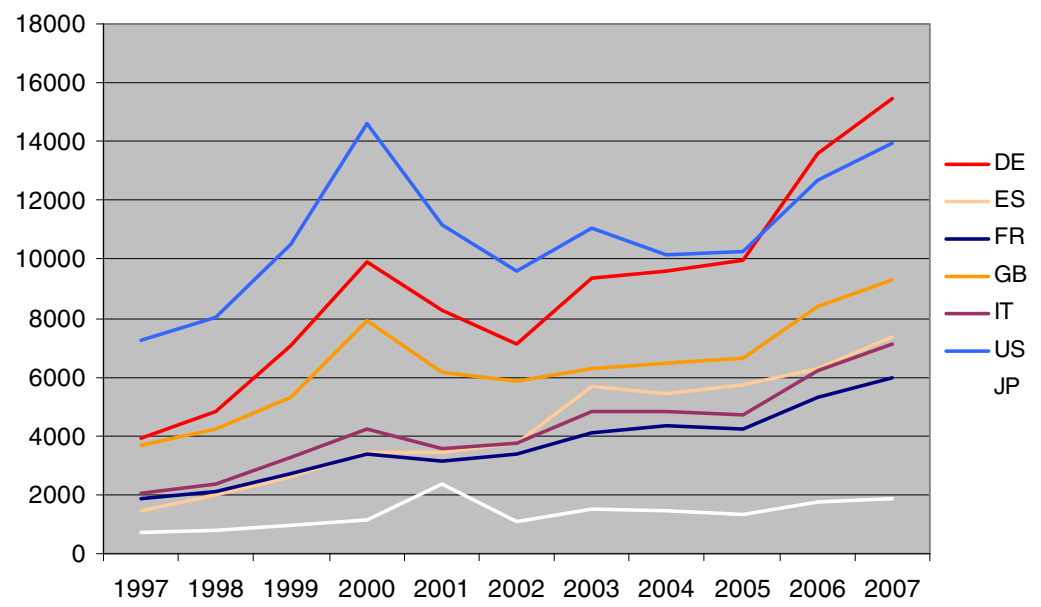

Fig. 2. Applications of CTMs for the largest applicant countries, absolute numbers, 1997-2007 ${ }^{1}$

Among non-EU countries US players' interest in the European-wide market is substantially higher than Japanese's, neither of which has yet surpassed their respective 2000-2001 levels where the "New Economy" bubble is clearly visible. Relative positions among the large economies are stable. But here comes a distinctive novelty: Germany has surpassed the US in 2006 as the top user of the CTM system, reaching unprecedented levels in 2007.

${ }^{1}$ OHIM data handed to the author from its central database, own calculations. See [3]. Note: Trends in absolute numbers are reported for large economies generating the highest volumes of applications: Germany (DE), United States (US), Great Britain (GB), Spain (ES), Italy (IT), France (FR), and Japan (JP). 
Trademark data also realizes its potential as an innovation indicator when combined with other, more conventional yardsticks of innovative performance. If we go back to the original definition by Schumpeter we realize that is emphasis on innovation as invention plus market introduction can operationalized using a proxy of technological capability (say R\&D or patents) and contrast that with the information provided by trademarks. Figure 3 uses country R\&D intensity (R\&D/GDP) on the $\mathrm{x}-$ axis and "trademark intensity" (CTMs/POP) on the y-axis to generate, for the first time, a picture of a possible taxonomy of countries. Although this can be seen as a crude mapping exercise it could be taken as producing some interesting results. We thus can see that there are countries above the average EU-15 both in technological and marketing capabilities (most Nordic countries), as well as those scoring bellow in both (most southern European countries). We can also see that Finland and France appear to be stronger on the technological front, whereas the Netherlands, Ireland and Spain have most of their relative strengths in marketing.

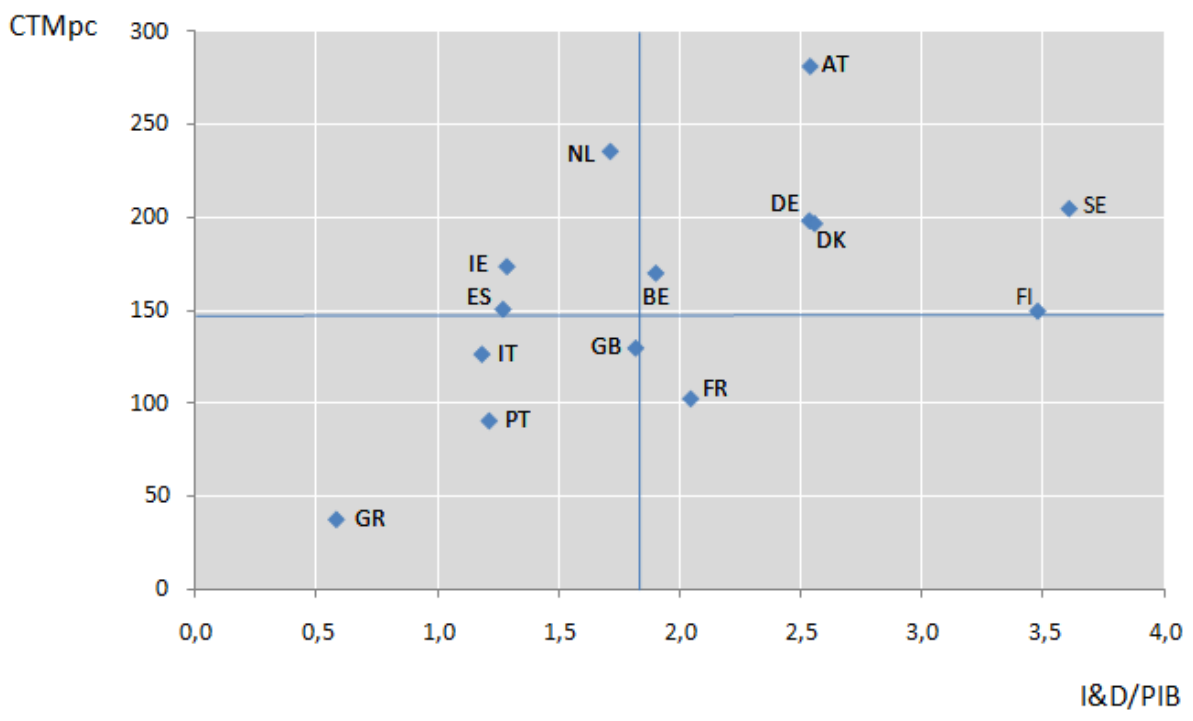

Fig. 3. Technological and marketing capabilities: a country taxonomy $[3]^{2}$

\section{Countries' Trademark-Intensiveness in Telecommunications}

Two classes are of interest as far as telecommunications are concerned, class 9 (containing a whole set of manufacturing goods, including scientific instruments and telecommunications equipment) and class 38 (a service class only referring to telecoms). As Table 1 shows both classes feature in the top 10 most trademarked classes.

${ }^{2}$ OHIM and OECD, own calculations. See [3]. Note. R\&D intensity = total expenditures in R\&D over GDP; Trademark intensity $=$ CTM application per million inhabitants. 
Table 1. Top classes in terms ${ }^{3}$

$\begin{array}{cl}\text { TOP-10 } & \text { World } \\ \mathbf{1} & \text { Instruments \& ICT } \\ \mathbf{2} & \text { Advertising and consultancy } \\ \mathbf{3} & \text { R\&D services } \\ \mathbf{4} & \text { Paper and packaging } \\ \mathbf{5} & \text { Education, culture, sports } \\ \mathbf{6} & \text { Clothing and footwear } \\ \mathbf{7} & \text { Telecoms } \\ \mathbf{8} & \text { Pharma and fine chemicals } \\ \mathbf{9} & \text { Light chemicals and cosmetics } \\ \mathbf{1 0} & \text { Finance }\end{array}$

\begin{tabular}{rlr}
\multicolumn{3}{c}{ EU-15 } \\
$\mathbf{1 0 \%}$ & Instruments and ICTs & $\mathbf{8 \%}$ \\
$\mathbf{7 \%}$ & Advertising and consultancy & $\mathbf{8 \%}$ \\
$\mathbf{7 \%}$ & R\&D services & $\mathbf{6 \%}$ \\
$\mathbf{6 \%}$ & Education, culture, sports & $\mathbf{6 \%}$ \\
$\mathbf{5 \%}$ & Paper and packaging & $\mathbf{5 \%}$ \\
$\mathbf{5 \%}$ & Clothing and footwear & $\mathbf{5 \%}$ \\
$\mathbf{4 \%}$ & Telecoms & $\mathbf{3 \%}$ \\
$\mathbf{4 \%}$ & Pharma and fine chemicals & $\mathbf{3 \%}$ \\
$\mathbf{3 \%}$ & Light chemicals and cosmetics & $\mathbf{3 \%}$ \\
$\mathbf{3 \%}$ & Leather and similar materials & $\mathbf{3 \%}$
\end{tabular}

Figure 4 isolates the two telecom-related tangible and intangible nice classes (respectively classes 9 and 38). These classes cover what could be broadly described and ICT/Telecom equipment and ICT/Telecom services. The telecoms data shows an upward trend punctuated by large fluctuations. The data reveals spikes around the year 2000 (the "New Economy" bubble) and a stagnation beyond 2007 (the "great recession"/"little depression").

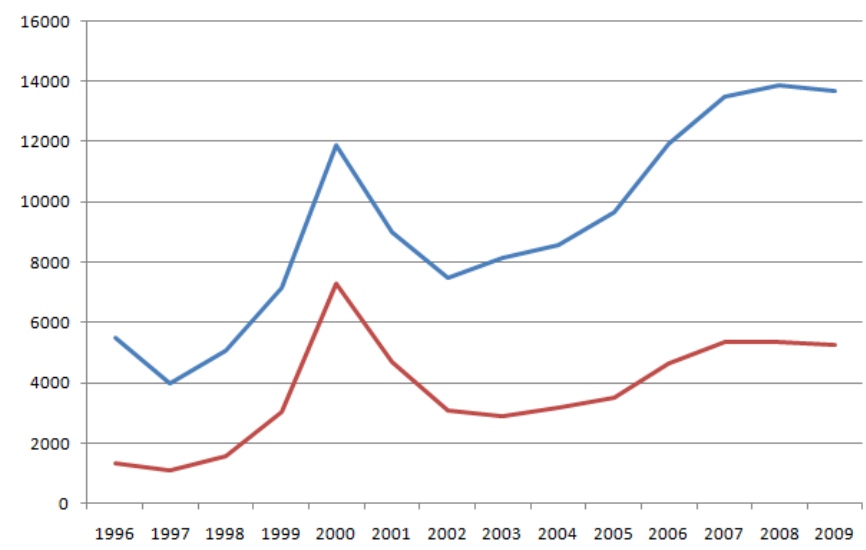

Fig. 4. New CTM applications in telecoms equipment (BLUE) and services (RED), 1997-2009³

Figure 5 analyses the concentration by country of new community trademark in telecommunications for the EU-15 in the period 1996-2009. Concentration decreases

${ }^{3}$ OHIM data, own calculations. For the use of trademarks as indicators of service innovation see [3], [4] and [5]. 
until the early 2000s but that dynamics seemed to have stopped by then. The indicator is the Hirshmann-Herfindahl index [3] and is defined as:

$$
I H H=\sum_{i=1}^{k} S_{i}^{2}
$$

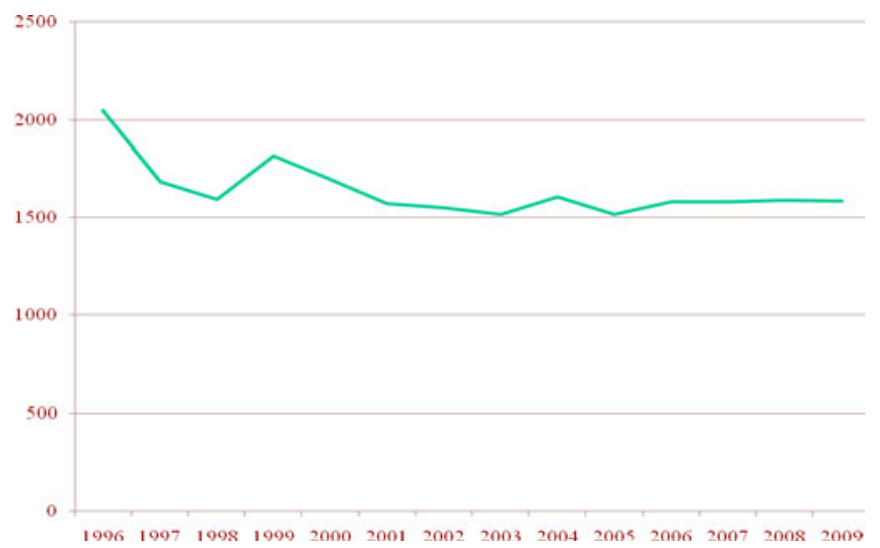

Fig. 5. Concentration in telecom services in the EU-15 (class 38), 1996-20094

The dynamic struggle for market shares is shown in Figure 6, which computes the instability index [3]. It also reinforces the notion that competition as been on the decrease for most of the time. The index is defined as:

$$
I I=\frac{1}{2} \sum_{i}^{n}\left|S_{i 2}-S_{i 1}\right|
$$

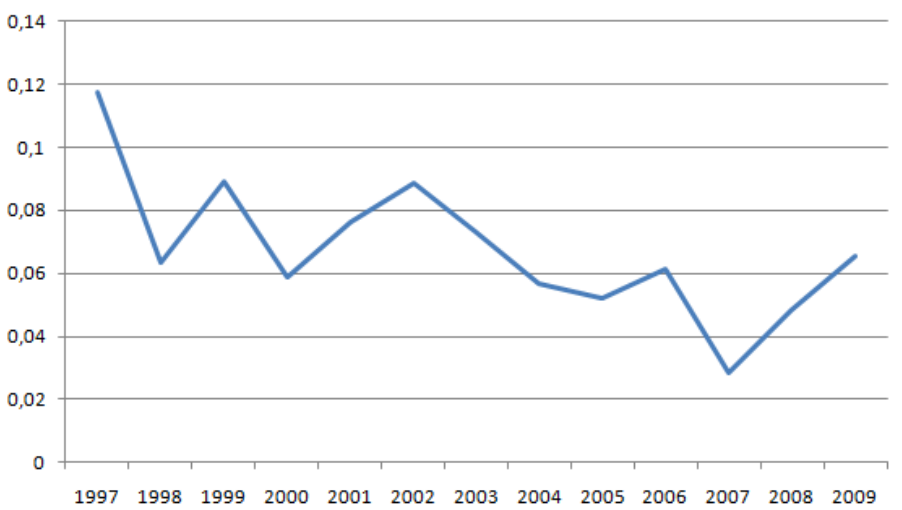

Fig. 6. Market instability in telecom services in the EU (class 38), 1996-2009 4

\footnotetext{
${ }^{4}$ OHIM data, own calculations. See [3].
} 
Figure 7 computes the "revealed marketing advantage" index for a sample of countries. Is a country is above one it has a competitive strength in a given product class. This approach allows us to put a taxonomy of countries. The index is:

$$
R M A_{i t}=\left[\frac{t m_{i t}}{\sum_{t} t m_{i t}}\right] / \frac{\sum_{i} t m_{i t}}{\sum_{i t} t m_{i t}}
$$

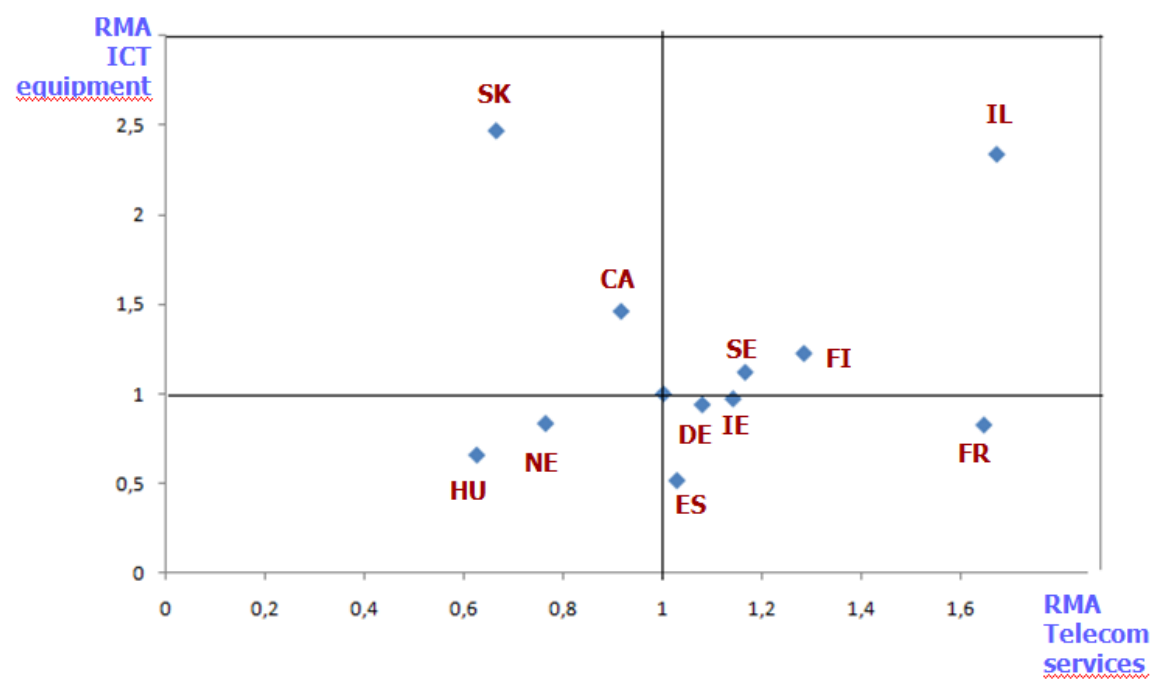

Fig. 7. Revealed marketing advantage in telecoms equipment and services ${ }^{5}$

\section{Conclusions}

Innovation does not consist exclusively of embodying technological knowledge into high-tech products. Innovation is wider a process that does not stop here; it also implies understanding, segmenting, targeting and persuading increasingly international customer bases of the uses of the new or improved, tangible or intangible artifacts. Thus, innovation is also about establishing the notoriety and credibility of products when introducing them in the marketplace. Typically reliant on technology-oriented indicators such as research and development (R\&D) or patents, empirical work on innovation has difficulty in capturing this marketing dimension of innovative activities. The goal of this section is to contribute to fill this gap by staying closely to Schumpeter's definition of innovation. It treats and measures innovation as the outcome of a combination of twin bodies of expertise: technological and marketing. We believe that trademarks are unique but still an under-exploited source of information for studying innovation behavior and industrial dynamics in the telecommunications sector. Thus, this potential should be utilized.

${ }^{5}$ OHIM data, own calculations. See [3]. 
Open Access. This article is distributed under the terms of the Creative Commons Attribution Noncommercial License which permits any noncommercial use, distribution, and reproduction in any medium, provided the original author(s) and source are credited.

\section{References}

1. Fagerberg, J.: Innovation: A guide to the literature. In: Fagerberg, J., Mowery, D., Nelson, R. (eds.) The Oxford Handbook of Innovation, pp. 1-26. Oxford University Press, Oxford

2. Mendonça, S., Pereira, T.S., Godinho, M.M.: Trademarks as an indicator of innovation and industrial change. Research Policy 33, 1385-1404 (2004)

3. Mendonça, S. (ed.): Estudo sobre o Contributo das Marcas para o Crescimento Económico e para a Competitividade Internacional. INPI (2011)

4. Schmoch, U.: Service marks as novel innovation indicator. Research Evaluation 12, 149-156 (2003)

5. Schmoch, U., Gauch, S.: Service marks as indicators for innovation in knowledge-based services. Research Evaluation 18(4), 323-335 (2009) 